\title{
NeuroImage
}

\section{Selective and interactive neural correlates of visual dimension changes and response changes}

\author{
Stefan Pollmann, ${ }^{\text {a,* }}$ Ralph Weidner, ${ }^{\mathrm{b}, \mathrm{c}}$ Hermann J. Müller, ${ }^{\mathrm{d}}$ \\ Marianne Maertens, ${ }^{a}$ and D. Yves von Cramon ${ }^{\mathrm{e}, \mathrm{f}}$ \\ a Department of Experimental Psychology, University of Magdeburg, Germany \\ ${ }^{\mathrm{b}}$ Research Center Jülich, Institute of Medicine, Germany \\ ${ }^{\mathrm{c}}$ Brain Imaging Centre West, Research Centre Jülich, Germany \\ ${ }^{\mathrm{d} D e p a r t m e n t ~ P s y c h o l o g y, ~ U n i v e r s i t y ~ o f ~ M u n i c h, ~ G e r m a n y ~}$ \\ ${ }^{\mathrm{e}}$ Day Clinic of Cognitive Neurology, University of Leipzig, Germany \\ ${ }^{\mathrm{f}}$ Max-Planck-Institute for Human Cognitive and Brain Sciences, Leipzig, Germany
}

Received 23 March 2005; revised 24 August 2005; accepted 7 September 2005

Available online 2 November 2005

In an event-related fMRI study, we investigated the neural correlates of visual dimension and response changes. We used a compound task, which required target selection by a singleton feature, a unique color or motion direction, before the appropriate motor response, which was determined by target orientation, could be selected. Both types of change elicited distinct patterns of activation, with dimension-changerelated activation primarily in posterior visual areas and responserelated activation primarily in motor-related areas of the parietal and frontal cortices. Response-change-related activation was delayed by about $1 \mathrm{~s}$ relative to dimension-change-related activation, suggesting that the latter is elicited by perceptual processes, whereas the former reflects response-related or post-response processes. Although dimension changes and response changes rely on different processes, they are not independent: response facilitation was observed for combined dimension and response repetitions, this facilitation, however, was disrupted by dimension changes.

(C) 2005 Elsevier Inc. All rights reserved.

\section{Introduction}

In visual search for singleton (odd-one-out) items, search costs are observed when the feature by which the target differs from the nontarget objects on a given trial is defined in a different visual dimension to that on the preceding trial (e.g., a color-defined, say red, target following a motion-defined target; Found and Müller, 1996). In contrast, no such change costs are observed when the target is defined by a different feature within the same dimension (e.g., a red target following a blue target). To explain this pattern of

* Corresponding author. Institut für Psychologie II, Otto-von-GuerickeUniversität, PF 4120, D-39016 Magdeburg, Germany. Fax: +49 39167 11947.

E-mail address: stefan.pollmann@nat.uni-magdeburg.de (S. Pollmann).

Available online on ScienceDirect (www.sciencedirect.com). results, Müller et al. (1995) proposed a 'dimension-weighting' account, according to which there is a limit to the total amount of attention, or attentional weight (cf. Bundesen, 1990; Duncan and Humphreys, 1989), available to be allocated to objects' dimensions. Potential target-defining dimensions (i.e., dimensions in which the target might differ from the nontarget objects) are assigned weight in accordance with their instructed importance and their variability across trials. Target detection requires that the target-defining dimension is weighted sufficiently to amplify the saliency (feature contrast) signal generated within this dimension above the detection threshold. Dimension changes incur a cost because attentional weight must be shifted from the old to the new dimension.

In previous event-related fMRI studies, we have investigated the neural network underlying these dimension-weighting processes. We identified an extended fronto-posterior network phasically activated when the target-defining dimension changed across trials (Pollmann et al., 2000b). This network consists of multiple posterior visual brain areas-including: fusiform gyrus, lateral occipital gyrus, superior temporal sulcus and middle temporal gyrus, superior parietal lobule, and precuneus - that are known to be involved in the attentional modulation of visual processing. In addition, we found prefrontal activations that have been related, primarily, to executive processes - in particular: in left frontopolar cortex and in anterior frontomedian cortex, at the anterior border of the pregenual anterior cingulate gyrus.

In the present study, we investigated the specificity of the dimension-weighting network by comparing stimulus-driven visual dimension changes with response changes. These issues are controversial. Recently, a debate has arisen in the behavioral literature as to whether dimension change effects are perceptual or, respectively, response-related in nature or whether they reflect both perceptual and response-related processes (see Müller and Krummenacher, in press, for a review). In more detail, Müller and his 
colleagues (e.g., Müller et al., 1995, 2003; Found and Müller, 1996; Krummenacher et al., 2001, 2002a) interpreted these effects as arising at a pre-attentive perceptual stage of processing that guides the allocation of focal attention to the target (based on preattentively computed feature difference signals). This interpretation has recently been challenged by Cohen (Cohen and Magen, 1999; Cohen and Feintuch, 2002; Feintuch and Cohen, 2002; Cohen and Shoup, 1997) and Theeuwes (Mortier et al., 2005; Theeuwes et al., in press; see also Theeuwes, 1992, 1996), who argued that the dimension change effects reflect post-selective response stage processes, which follow the allocation of focal attention to the singleton feature target (with attentional allocation itself being uninfluenced by dimension weighting). The present fMRI study was designed to dissociate perceptual from response-related processes in dimensional weighting in order to broaden the data base for making a decision among the various theoretical alternatives.

To investigate this issue, the present study employed a visual singleton search paradigm using a 'compound' task (in terms of Duncan, 1985), in which the target, a triangle stimulus pointing left or right, to be detected among nontarget triangles was characterized by a salient feature in one of two possible dimensions, color or motion, whereas the response to be made was governed by the target's pointing direction, left or right (Fig. 1). For instance, an odd-one-out target could be a single red triangle within a display of
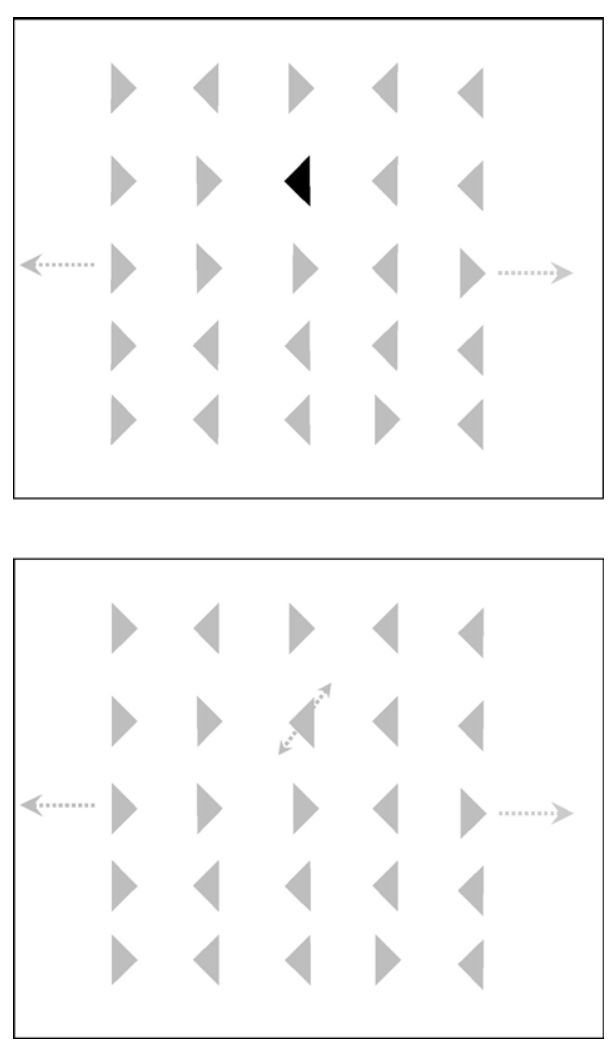

Fig. 1. Search displays. Search displays consisted of a matrix of $(5 \times 5) 25$ triangles each pointing randomly to the left or right. In each display, one triangle, the target, differed from the others, the distractors: either by its color (red as compared to green) or its direction (axis) of sinusoidal motion ( $45^{\circ}$ oblique as compared to horizontal). Red is indicated by black and green by gray in the figure. Observers had to detect the odd-one-out target triangle and make a button press indicating the target's pointing direction. green triangles, all triangles moving uniformly (sinusoidally) in horizontal direction (color-defined target). On the next trial, the target could be a green triangle (among green nontarget triangles) moving in a unique direction: oblique rather than horizontal (motion-defined target). Thus, target detection depended on finding a salient color or, respectively, motion feature difference. In contrast, the response was governed by the pointing direction of the target triangle: if it pointed to the left or the right, the left or, respectively, the right one of two buttons had to be pressed. The nontarget triangles pointed randomly to the left and right. Changes in the target-defining dimension across trials, such as from color to motion (as in the above example), occurred orthogonally to changes in the required response.

We expected visual dimension changes to elicit a phasic increase of the fMRI signal in posterior visual brain areas, as well as anterior areas in parietal and frontal cortex that have previously exhibited dimension-change-related activation and may support shifts of attentional weight from the old to the new target-defining dimension (Pollmann et al., 2000b; Weidner et al., 2002). In contrast, a response-change-related signal increase was expected in motor cortex, as well as in brain areas involved in linking the critical stimulus, the (changed) pointing direction of the target triangle, to the appropriate response, such as the anterior intraparietal area (AIP) and premotor areas involved in (re-)programming the response. Response change trials may trigger a series of cognitive processes: discriminating the target triangle's (changed) pointing direction, inhibiting the response primed by the previous trial, preparing the new response, and eliciting this response. The current experiment was not designed to discriminate between these processes; rather, its intention was to separate visual dimensionchange-related activation changes from response-related processes in general.

In addition, the present study asked whether the effects of visual dimension changes and of response changes would interact at some point in the processing, which may be expected from the behavioral literature (e.g., Hommel, 1998; Kingstone, 1992; Lockhead et al., 1978).

\section{Methods \\ Participants}

Twenty-one observers took part in a single fMRI experimental session. One of them was excluded from the analysis because a large frontal signal void due to susceptibility artefacts reached into the frontopolar region that we had found active during dimension changes in previous studies. The remaining 20 participants (9 female) ranged between 21 and 37 years in age, with a mean age of 26.4 years. All observers were right-handed, as assessed by the Edinburgh Handedness Inventory (Oldfield, 1971). The fMRI procedures were approved by the University of Leipzig ethics committee. All observers gave prior written informed consent according to the guidelines of the Max Planck Institute.

\section{Stimuli, task, design, and procedure}

Stimuli were displayed by an LCD projector on a backprojection screen mounted in the bore of the magnet behind the observer's head. Observers viewed the screen wearing mirror glasses, which were equipped with corrective lenses if necessary. 
The fMRI session began with the presentation of a 30-s fixation period, followed by the presentation of 624 experimental trials, and ending with a 30 -s fixation period. Trial duration was $1.5 \mathrm{~s}$. Each trial began with the presentation of a search display. The display was terminated by the observer's response or after a maximum duration of $1.5 \mathrm{~s}$. A white fixation cross was displayed during the inter-trial interval, which lasted, variably, 0, 500, or $1000 \mathrm{~ms}$. Each of the four experimental conditions was presented with a probability of 0.2 , the remaining trials were null events. The order of events, including null events, was determined using maximumlength shift register sequences ( $m$-sequences) described in detail by Buracas and Boynton (2002). M-sequences counterbalance subsequences of a given length in order to ensure that trials from each condition were preceded equally often by trials from each of the other conditions.

The visual search displays consisted of 25 triangles on a black background. The stimuli were arranged in a grid-like pattern, covering an area of $13^{\circ} \times 13^{\circ}$ of visual angle. Each triangle pointed randomly in one of two directions, equally often to the left or to the right (Fig. 1). All stimuli moved sinusoidally along the horizontal axis (maximum amplitude $=0.2^{\circ}$, speed $=1.2^{\circ} \%$ ). Each search display contained a singleton pop-out target, which was equally likely to be defined by a unique color relative to the nontargets (a red horizontally moving triangle among green horizontally moving triangles) or by a unique motion direction (a green triangle moving along an oblique axis tilted oriented $+45^{\circ}$ from the horizontal among horizontally moving green triangles).

Observers were asked to give a speeded forced-choice response indicating the pointing direction of the target triangle, using their right-hand index (left button) or middle finger (right button), respectively.

\section{fMRI measurement}

Functional images were collected at $3 \mathrm{~T}$ by a Bruker 30/100 Medspec system (Bruker Medizintechnik, Ettlingen, Germany), using a gradient echo EPI sequence $(\mathrm{TR}=2000 \mathrm{~ms}, \mathrm{TE}=30 \mathrm{~ms}$, flip angle $=90^{\circ}$ ). Twenty axial slices were acquired parallel to the AC-PC plane, allowing for whole brain coverage. Slice thickness was $4 \mathrm{~mm}$ and inter-slice distance $1 \mathrm{~mm}$, with a 19.2-cm FOV and a $64 \times 64$ image matrix. Data were analyzed using the LIPSIA software package (Lohmann et al., 2001). Movement artefacts were corrected using a matching metric based on linear correlation. Slice acquisition time differences were corrected by sinc interpolation. Baseline drifts were corrected by high-pass filtering, implemented using a discrete Fourier transform with an individually tailored cut-off period of five times the mean temporal distance between trials of the same experimental condition. In the spatial domain, the data were filtered using a Gaussian filter with FWHM $=7 \mathrm{~mm}$. Following this preprocessing, the functional data sets were co-registered with the individual observers' high-resolution anatomical data sets and normalized by linear scaling. The normalized data set had a resolution of $3 \times$ $3 \times 3 \mathrm{~mm}$ voxels. Data were analyzed using the general linear model (Friston et al., 1995). Event-related analyses were performed using a combination of parameter estimates of a basis set of gamma functions (as implemented in SPM2; Fig. 2). This procedure makes fewer assumptions about the shape of signal changes than modeling using a canonical hemodynamic response function (indeed, preliminary analyses using the latter procedure revealed that many signal changes were not well modeled by a canonical hemodynamic response function). We contrasted (1) the condition-specific beta-estimates of the first gamma function to capture phasic early onset signal changes, (2) the linear combination of the beta-estimates of the first and second gamma function, and (3) the linear combination of all three gamma functions to capture signal changes of gradually increasing peak latency and duration. All areas that were revealed to be significant in these contrasts are listed in Table 1. Group activation was calculated using a random-effects model (Holmes and Friston, 1998). Significance threshold for whole-brain analyses was $\alpha=$ 0.0001 . Only activations with a minimum cluster size of $81 \mathrm{~mm}^{3}$ (equivalent of 3 contiguous $3 \times 3 \times 3 \mathrm{~mm}$ voxels obtained after normalization) were considered to reduce the probability of false positives. At the chosen level, the corrected $\alpha$ level for our data set was estimated to be less than $P=0.05$ for the family of three tests performed independently with different (combinations of) gamma functions (Forman et al., 1995). We have previously

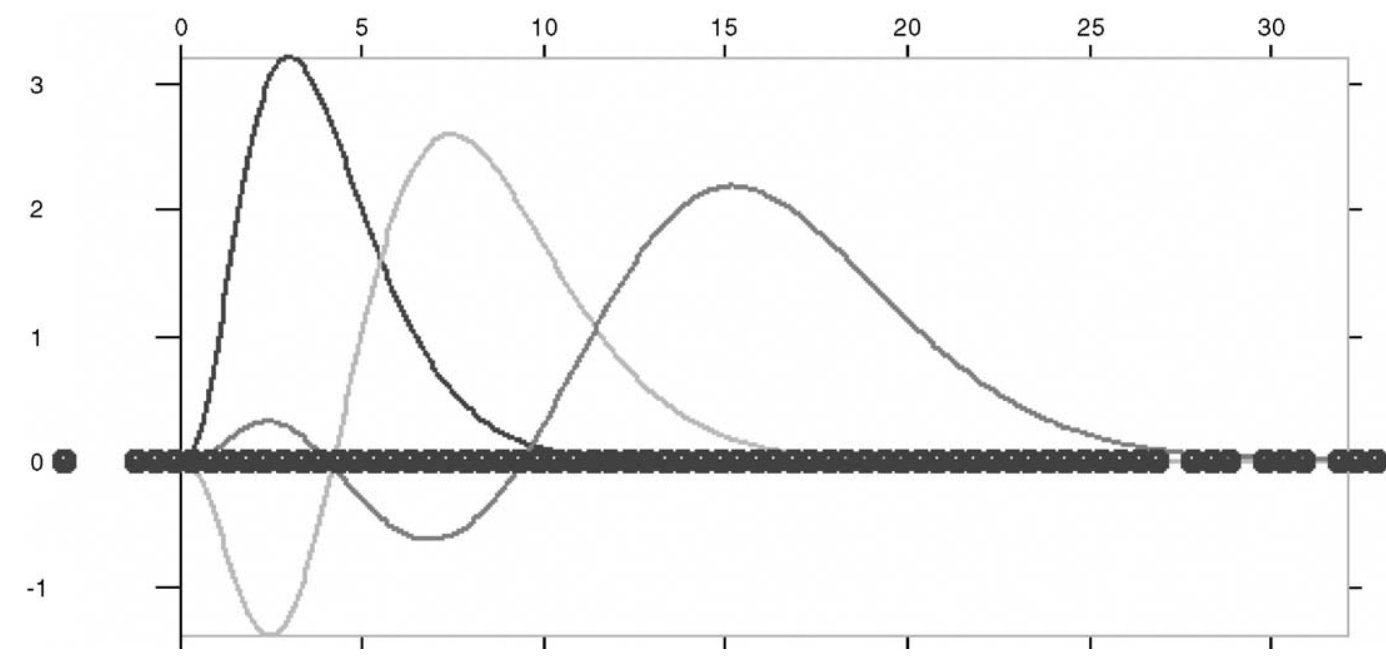

Fig. 2. Basis set of gamma functions. The BOLD response was modeled with this basis set of three gamma functions. Early phasic signal increases were modeled with parameter estimates of the first gamma function alone. Longer lasting signal changes were modeled by linear combinations of the parameter estimates of the first two or all three gamma functions. The $x$ axis indicates time in seconds, the $y$ axis size in arbitrary units. 
Table 1

List of activations

\begin{tabular}{|c|c|c|c|c|c|c|c|c|}
\hline Extent $\left(\mathrm{mm}^{3}\right)$ & Zmax & Hemi & $x$ & $y$ & $z$ & Structure & Model & ROI-Nr. \\
\hline \multicolumn{9}{|c|}{ Dimension change $>$ dimension stay } \\
\hline 140 & 3,5 & $\mathrm{~L}$ & -34 & -43 & 35 & $\begin{array}{l}\text { intraparietal sulcus } \\
\text { (horizontal) }\end{array}$ & $\mathrm{g} 1$ & 1 \\
\hline 83 & 3,38 & $\mathrm{R}$ & 26 & -73 & 18 & intraoccipital sulcus & g1 & 2 \\
\hline 542 & 3,57 & $\mathrm{R}$ & 26 & -76 & -3 & fusiform gyrus & $\mathrm{g} 1$ & 3 \\
\hline 85 & 3,31 & $\mathrm{R}$ & 19 & -85 & 6 & striate/peristriate cortex & g1 & 4 \\
\hline 149 & 3,78 & $\mathrm{R}$ & 28 & -31 & 9 & posterior putamen/claustrum & g1 & 5 \\
\hline \multicolumn{9}{|c|}{ Response change $>$ response stay } \\
\hline 219 & 3,64 & $\mathrm{~L}$ & -41 & -37 & 47 & intraparietal sulcus (ascending) & g1 & 6 \\
\hline 243 & 3,69 & $\mathrm{~L}$ & -65 & -40 & 9 & superior temporal gyrus & g1 & 7 \\
\hline 161 & 3,6 & $\mathrm{~L}$ & -23 & 17 & 59 & $\begin{array}{l}\text { superior frontal sulcus } \\
\text { (middle portion) }\end{array}$ & g2 & 8 \\
\hline 140 & 3,52 & $\mathrm{~L}$ & -23 & 14 & 30 & inferior frontal sulcus & g2 & 9 \\
\hline 241 & 3,76 & $\mathrm{~L}$ & -34 & -19 & 59 & precentral gyrus & $\mathrm{g} 2$ & 10 \\
\hline 97 & 3,64 & $\mathrm{~L}$ & -46 & -64 & 44 & angular gyrus & g2 & 11 \\
\hline 178 & 3,9 & $\mathrm{~L}$ & -35 & -37 & 0 & hippocampal formation & g2 & 12 \\
\hline 268 & 4,19 & $\mathrm{R}$ & 13 & -55 & -12 & Cerebellum & g2 & 13 \\
\hline 394 & 3,99 & $\mathrm{~L}$ & -43 & -70 & -27 & Cerebellum & g2 & 14 \\
\hline 114 & 3,61 & $\mathrm{R}$ & 25 & -76 & -27 & Cerebellum & g2 & 15 \\
\hline 86 & 3,51 & $\mathrm{~L}$ & -20 & 20 & 32 & $\begin{array}{l}\text { superior frontal sulcus } \\
\text { (middle portion) }\end{array}$ & g3 & 16 \\
\hline 360 & 4,18 & $\mathrm{~L}$ & -35 & -13 & 62 & precentral gyrus & g3 & 17 \\
\hline 1332 & 3,98 & $\mathrm{~L}$ & -47 & -25 & 53 & postcentral gyrus & g3 & 18 \\
\hline 197 & 4,08 & $\mathrm{~L}$ & -23 & -49 & 27 & parieto-occipital fissure & g3 & 19 \\
\hline 89 & 3,35 & $\mathrm{R}$ & 20 & -55 & -15 & Cerebellum & g3 & 20 \\
\hline \multicolumn{9}{|c|}{ Interaction dimension change $\times$ response change } \\
\hline 95 & 3,8 & $\mathrm{R}$ & 22 & -55 & 24 & parieto-occipital fissure & g2 & 21 \\
\hline
\end{tabular}

Coordinates are according to Talairach and Tournoux (1988), Zmax: maximal $z$ value, hemi: hemisphere, L/R: left/right hemisphere. The 'Model' column indicates the parameters used for the contrast: g1: parameter of first gamma function from the basis set (Fig. 2). g2: linear combination of parameter estimates for first and second gamma function, g3: linear combination for all three gamma functions (see Methods for details). ROI numbers correspond to numbering in Figs. 4 and 5 .

shown that rapid event-related designs, such as the one used here, permit the differential response between experimental conditions to be measured without amplitude loss due to BOLD (blood oxygenation level dependent) response overlap across successive trials (Pollmann et al., 2000a).

Because of the flexibility of this modeling approach, knowledge of the signal time course is a prerequisite for a functional interpretation. Time courses of the BOLD signals were extracted for selected activations at the location of the maximum group activation. The standard error of the means was calculated by jackknife resampling (Efron, 1977; Ruge et al., 2003).

\section{Results}

\section{Behavioral data}

Fig. 3 presents the group mean reaction times (RTs) as a function of dimension and response changes. A repeated-measures ANOVA of the RT data, with the factors dimension change (change, no change) and response change (change, no change), revealed only the interaction to be significant $\left(F(1,19)=6.9, \mathrm{MS}_{\mathrm{e}}=2266, P<0.05\right)$. Collapsed across response change conditions, there was no significant RT increase for trials on which the target dimension changed (relative to the preceding trial) compared to no-change trials: 718 versus $714 \mathrm{~ms}$ (non-significant main effect of dimension change, $\left.F(1,19)=2.3, \mathrm{MS}_{\mathrm{e}}=234.14, P>0.05\right)$. Similarly, RTs, collapsed across dimension change conditions, were not significantly increased for response change compared to no-change trials, 719 versus $713 \mathrm{~ms}$ (non-significant main effect of response change, $\left.F(1,19)=2.7, \mathrm{MS}_{\mathrm{e}}=613.0, P>0.05\right)$.

In the absence of a response change, changes in the target dimension significantly slowed RTs $(720$ and $706 \mathrm{~ms}$ for dimension change and no-change trials, respectively; $t(19)=2.6$, $P<0.05$ ); in contrast, when there was a response change, RTs tended to be faster when the target dimension changed, too (715 and $722 \mathrm{~ms}$, respectively; $t(19)=1.95, P=0.07)$. In the absence of a dimension change, changes in the response significantly slowed RTs (722 and $706 \mathrm{~ms}$ for response change and no-change trials, respectively; $t(19)=3.1, P<0.01$ ); however, when there was a dimension change, RTs were somewhat, though not significantly, faster when the response changed as well $(715$ and $720 \mathrm{~ms}$ for response change and no-change trials, respectively; $t(19)=0.95$, $P>0.05)$. This interactive pattern of dimension change and response change effects is robust: the same pattern was observed in a reanalysis of the compound task RT data of Krummenacher et al. (2002b) and Müller and Krummenacher (in press).

Error rates were low overall $(3.1 \%)$. In order to rule out that the RT effects were due to speed-accuracy trade-offs, the error data were examined by an analogous dimension change (change, no change) $\times$ response change (change, no change) ANOVA. This ANOVA revealed only the interaction to be significant (dimension 


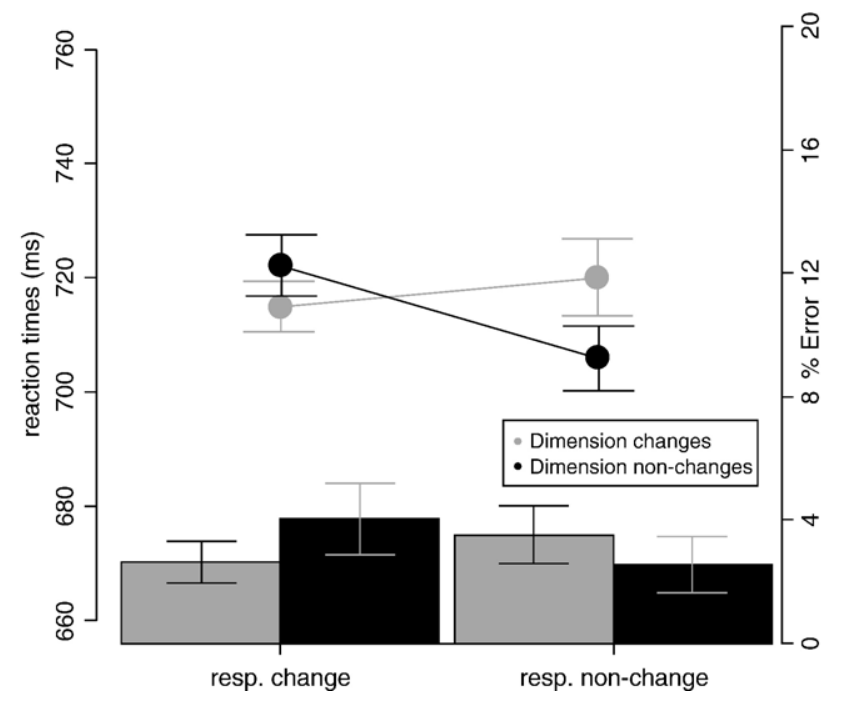

Fig. 3. Reaction times and error rates in the fMRI experiment as a function of dimension and response changes. Error bars indicate the $95 \%$ confidence intervals (CI) corrected for inter-individual differences (Loftus and Masson, 1994).

change: $F(1,19)=0.5261, \mathrm{MS}_{\mathrm{e}}=0.009$, n.s; response change: $F(1,19)=0.1162, \mathrm{MS}_{\mathrm{e}}=0.017$, n.s.; dimension change $\times$ response change: $\left.F(1,19)=13.105, \mathrm{MS}_{\mathrm{e}}=0.28, P<0.01\right)$. When there was no response change, there was a tendency towards higher error rates on dimension change compared to no-change trials $(3.5 \%$ versus $2.5 \%, t(19)=0.77, \mathrm{n} . \mathrm{s})$, while in the presence of response change, there was a tendency towards lower error rates on dimension change trials $(2.6 \%$ vs. $4.1 \%, t(19)=0.59$, n.s $)$. This pattern of error effects reinforces the RT effects.

\section{Functional imaging}

\section{Dimension changes}

The functional imaging data were analyzed analogously to the behavioral data. Increased dimension-change-related activation was analyzed by the main effect of dimension change across conditions of response change. Increased activation was observed in occipital and parietal cortex, predominantly in the right hemisphere (Table 1). In occipital cortex, activations were observed in right striate/ peristriate cortex and in right fusiform gyrus (Figs. 4a, b). Further activations were located at the termination of the right intraoccipital sulcus (Fig. 4a) and along the horizontal segment of the left intraparietal sulcus. Finally, an activation was located in the posterior putamen, bordering the claustrum.

We modeled signal changes of different latencies and durations by means of linear combinations of the parameter estimates of a basis set of gamma functions (see Methods for details). All dimension-change-related activations showed early phasic signal increases, as indicated by a significantly increased beta-weight of the first gamma function (Fig. 2). No significant differences were observed when we modeled temporally more extended responses (by linear combination of the first two or all three gamma functions; Table 1).

The signal time courses for selected areas are presented in Fig. 4. Dimension changes, compared to non-changes, showed an early signal increase, which peaked after $3 \mathrm{~s}$ along the intraoccipital sulcus (Fig. 4a) and in fusiform gyrus (Fig. 4b). Note that these are relative signal changes between dimension change and no-change trials; that is, a peak after $3 \mathrm{~s}$ indicates that the difference between change and no-change trials was maximal after $3 \mathrm{~s}$ (and not that the BOLD response to dimension changes peaked after $3 \mathrm{~s}$; the BOLD responses for all conditions are presented in the Appendix A). In all these areas, signal increases for dimension changes versus response changes (indicated by the non-overlapping standard deviations) were observed within an early time window from 1 to $4 \mathrm{~s}$ after stimulus onset.

\section{Response changes}

Increased response-change-related activation was analyzed by the main effect of response change, across conditions of dimension change. Increased activation for response changes was observed in an extended network of cortical and subcortical areas. In contrast to dimension changes, response changes elicited mostly later and longer-lasting BOLD response changes than visual dimension changes, indicated by significant changes in the linear combinations of the first and second or all three gamma functions (Table 1).

In left motor cortex, response changes elicited a signal increase in the middle genu of the left precentral gyrus at the location of the hand motor representation area (Yousry et al., 1997; Fig. 5c). Several activation loci clustered around the hand area, one immediately anterior, in the precentral gyrus bordering the precentral sulcus (Fig. 5b), and an extended activation in postcentral gyrus, immediately posterior to the motor hand area (Fig. 5d). More anteriorly, an activation was observed along the left superior frontal sulcus (Fig. 5a).

In parietal cortex, response changes elicited increased activation in the left ascending segment of the intraparietal sulcus (Fig. 4c), in the left angular gyrus, and in the fundus of the left parieto-occipital fissure. Further signal increases were observed in the hippocampal formation and the cerebellum.

In primary motor cortex (MI; Fig. 5c), premotor cortex (Fig. $5 \mathrm{~b}$ ), and primary sensorimotor cortex (SI, Fig. 5d), response changes elicited a signal increase which started about $2 \mathrm{~s}$ and lasted until about $10 \mathrm{~s}$ after stimulus onset, with minor variations between areas. Interestingly, response changes elicited a rather late signal increase peaking $8 \mathrm{~s}$ post-stimulus onset in the anterior part of left middle frontal gyrus, bordering the superior frontal sulcus (Fig. 5a). In the cerebellum, response changes went along with a higher BOLD response from 5 to $11 \mathrm{~s}$, with a peak at $8 \mathrm{~s}$, post-stimulus onset (Fig. 5e).

Across areas, we observed an early response-change-related peak at $4 \mathrm{~s}$ and a later peak at $8 \mathrm{~s}$ post-stimulus onset. The early peak was observed in left lateral premotor cortex (Fig. 5b), left SI (Fig. 5d), and the left ascending segment of the intraparietal sulcus (AIP; Fig. 4c). The later peak was observed in left middle frontal gyrus and the left cerebellar hemisphere as the main peak and in left lateral premotor cortex as a second peak.

\section{Timing of dimension-change- and response-change-related activation}

In general, dimension-change-related signal increases seemed to have an earlier onset and offset and a shorter range than response-related signal increases. To examine for these time differences, we conducted one-tailed independent samples $t$ tests on the group signal time courses for the regions shown in Figs. 4 and 5. Differences in signal time course parameters across areas may be of vascular origin as much as reflecting task-related 
a)

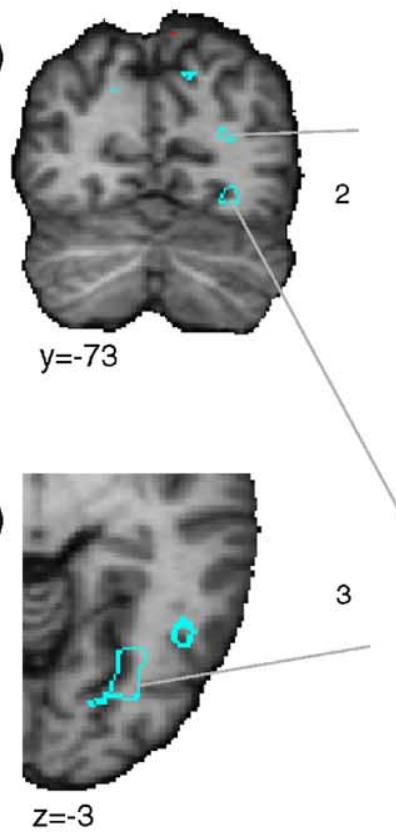

c)

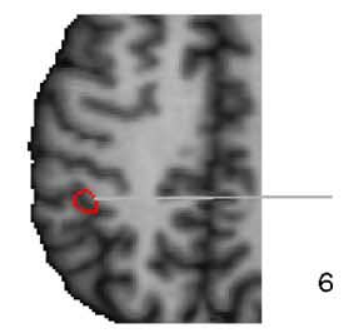

$\mathrm{Z}=47$

d)

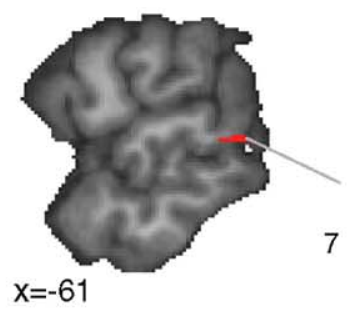

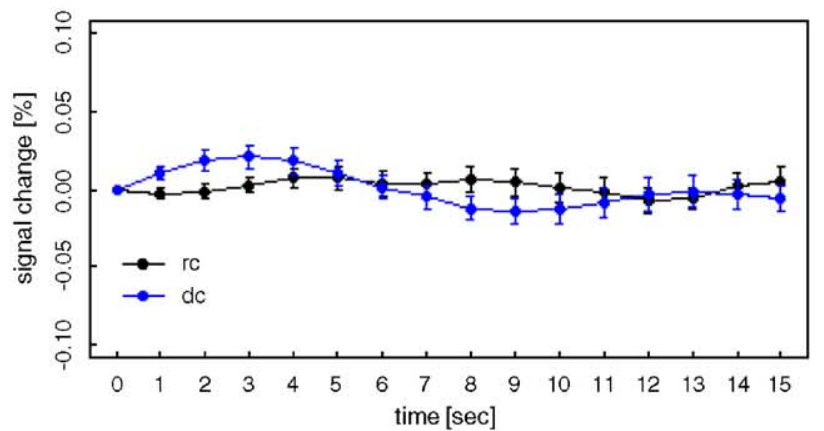
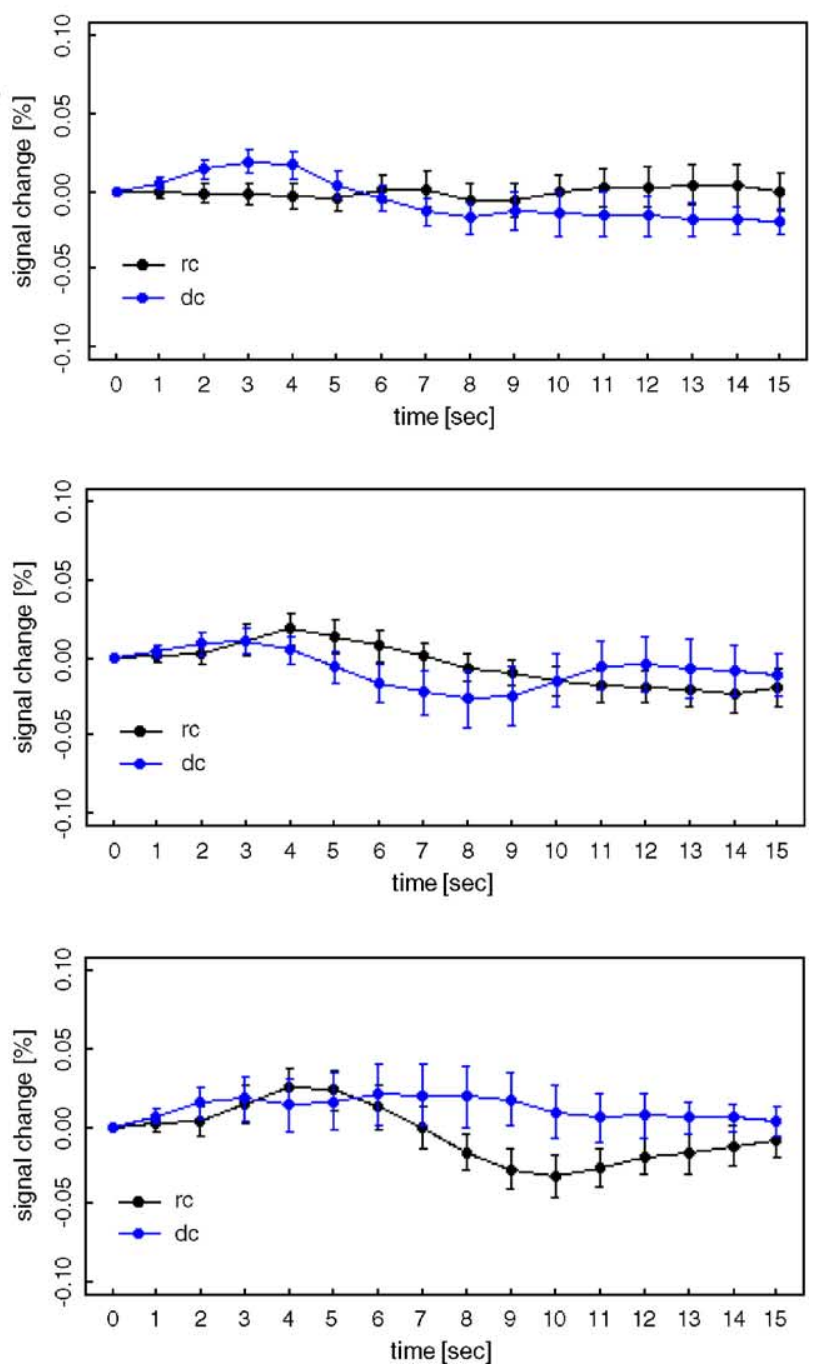

Fig. 4. Early dimension-change- and response-change-related signal changes. Dimension changes elicited early signal increases, identified by a significant increase in the beta-value of the first gamma function (see Methods for details), were observed at the end of the right intraoccipital sulcus (a) and in the right fusiform gyrus (b). Response changes elicited early phasic activations at the banks of the ascending left intraparietal sulcus (c) and in the left superior temporal gyrus, bordering the superior temporal sulcus (d). Colors indicate significant dimension-change- (turquoise) and response-change-related activation (red). The time courses represent the averaged differential event-related signal time courses for the following contrasts: dc: dimension change-dimension repetition trials, rc: response change-response repetition trials. Error bars indicate the standard errors of the means, obtained by jack-knife resampling. The image planes are identified by the appropriate coordinates of the Talairach and Tournoux (1988) system. For full coordinates, see Table 1.

processing differences. In order to minimize this potential confound, we compared signal latencies in multiple areas with dimension-change-related activation with multiple areas with response-change-related activations. Pooling across areas reduces this possible confound but does not eliminate it. Thus, these results need to be interpreted with caution.

Onsets and offsets were defined as the first and last points in time at which the dimension-change- and, respectively, response- 
a)

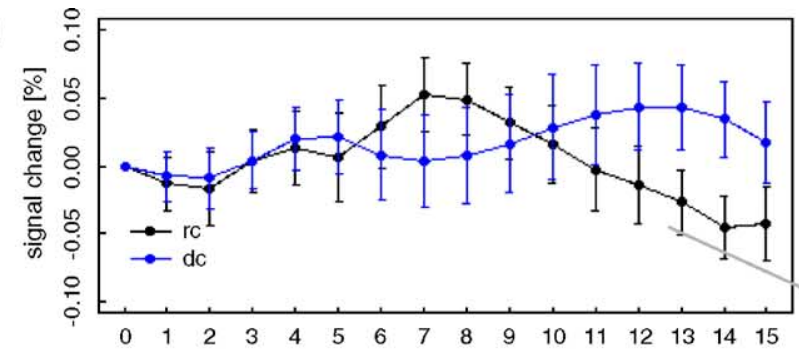

b)
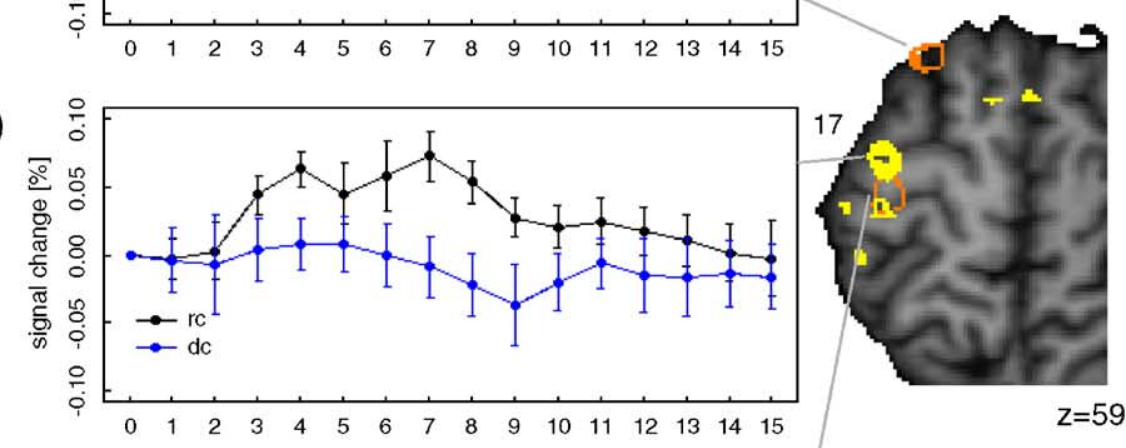

c)

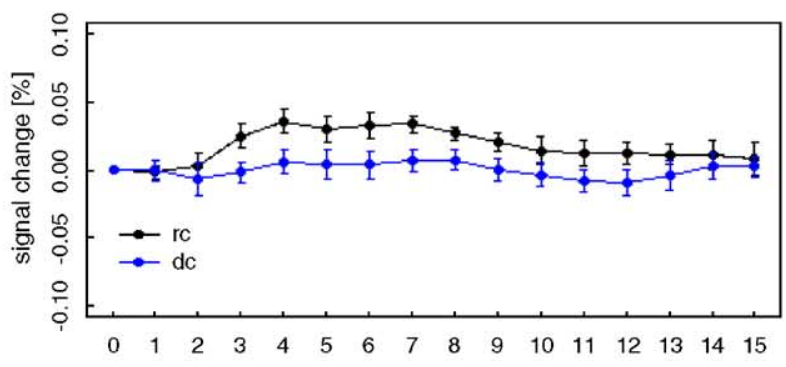

10

8

d)

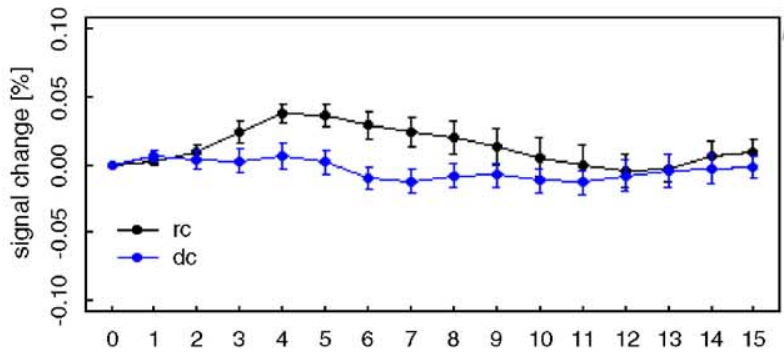

e)

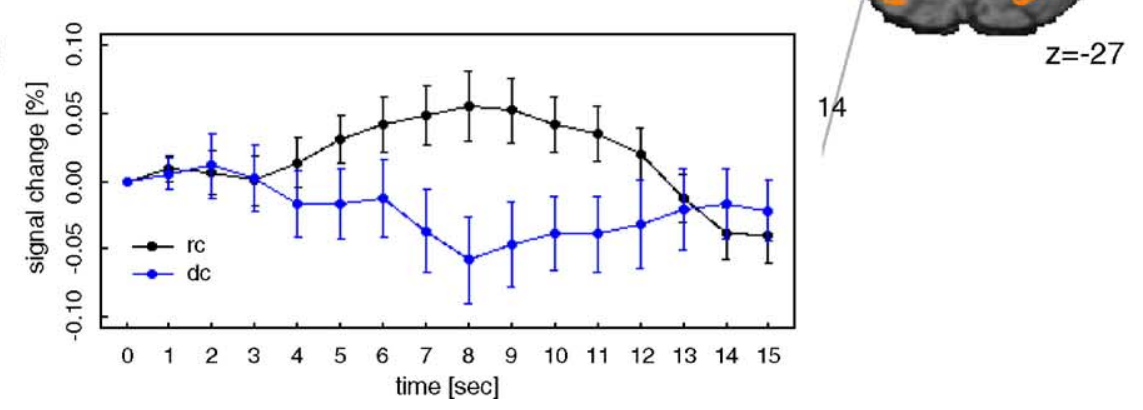

Fig. 5. Response-change-related activation of longer duration. Activations of longer duration were identified by significantly increased linear combinations of the beta-estimates of the first and second gamma functions (shown in orange) or all three gamma functions (shown in yellow). See Fig. 4 for abbreviations.

change-related signals were more than one standard error of the mean above zero. Levene tests revealed unequal variances for offsets and ranges; degrees of freedom were adjusted accordingly. Significance criterion was $\alpha=0.05$. Tests confirmed earlier onsets $(t=2.67, d f=8, P<0.05)$ and offsets $(t=3.94, d f=6, P<0.05)$ for dimension-change-related signal increases. Range did not differ significantly $(t=1.66, d f=6, P>0.05)$.
Interaction between dimension and response changes

A significant interaction between dimension and response change was observed at the fundus of the right parieto-occipital fissure. Dimension changes were accompanied by differential activation relative to non-changes only when no response changes were required (Fig. 6). In this case, dimension repetitions led to higher activation than dimension changes. Response changes did 


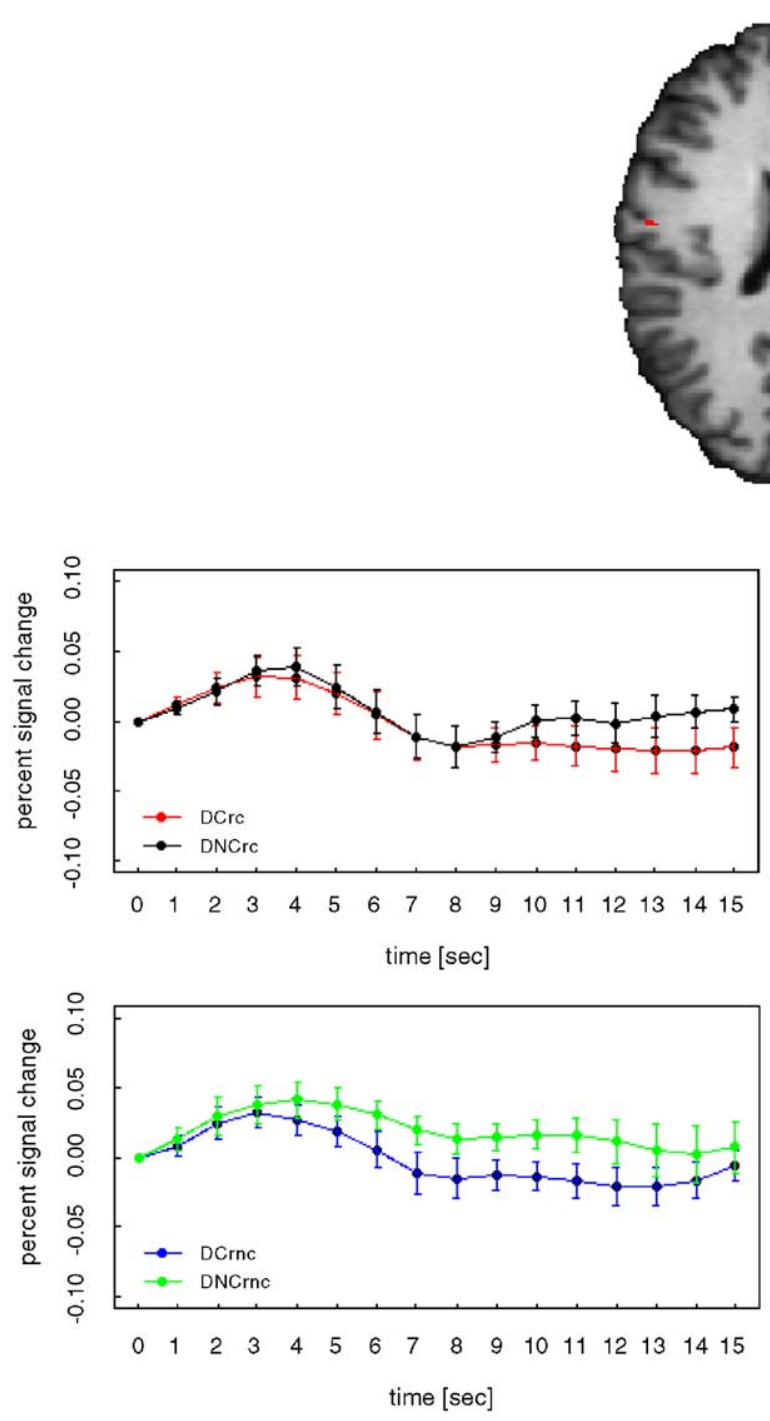

Fig. 6. Interaction of dimension-change- and response-change-related activation. A region at the fundus of the parieto-occipital fissure showed an interaction between dimension-change- and response-change-related activation. $\mathrm{D}(\mathrm{N}) \mathrm{C}$ : dimension (non) change, $\mathrm{r}(\mathrm{n}) \mathrm{c}$ : response (non) change.

not lead to differential activation, irrespectively of whether or not they were accompanied by dimension changes. This is the mirror image of the increased dimension-change-related activation observed in posterior brain areas. Furthermore, it is worth noting that peak amplitudes for dimension repetitions at the fundus of the parieto-occipital fissure were reached $4 \mathrm{~s}$ after stimulus onset, resembling more the response-change- than the dimensionchange-related activation.

\section{Discussion}

\section{Dimension change}

The dimension weighting account (Müller et al., 1995; Found and Müller, 1996) proposes that, in the case of a dimension change, attentional weight is reallocated from the old to the new targetdefining dimension. The current activation pattern supports this view: there was a large activation in right fusiform gyrus, at a location that has previously been reported to be a potential source of the P1 event-related electric brain potential, which itself is modulated by selective attention (Heinze et al., 1994). Likewise, the cortex at the banks of the intraoccipital sulcus, where we found increased activation following dimension changes, has also been reported to be modulated by selective attention in an fMRI study and to be a second potential source of the P1 (Martinez et al., 1999; separate ventral and dorsal P1 sources may be due to the retinotopic organization of higher-tier visual cortices).

In an additional analysis, to be reported in a separate paper, we observed increased activation in right posterior fusiform gyrus when color, rather than motion, was the target-defining dimension (Pollmann et al., in press). The reverse pattern, increased activation during motion, compared to color, epochs, was observed in right lateral occipital gyrus. The fusiform signal increase during color epochs was located somewhat posteriorly to the location of posterior V4 (as specified in Bartels and Zeki's, 2000, review of color processing studies). The signal increase during motion-defined epochs agreed well with previous reports of the location of hMT+. This further supports the notion that dimension changes lead to shifts of attention between dimension-specific visual input areas. 
Dimension changes further elicited activation along the descending and horizontal segments of the intraparietal sulcus, whereas response changes led to activation in its ascending segment. This distribution is in good agreement with studies of visual attention shifts, both between locations and between features, which have consistently reported activation along the horizontal segment of the intraparietal sulcus (Corbetta et al., 1998; Gitelman et al., 1999; Lepsien and Pollmann, 2002; Liu et al., 2003; Pollmann and von Cramon, 2000; Pollmann et al., 2000b; Weidner et al., 2002; Yantis et al., 2003), and with studies of prehensile movements (Binkofski et al., 1998, 1999); and it is consistent with studies of motor attention (Rushworth et al., 2001), which have reported activation along the ascending segment of the intraparietal sulcus and functional deficits following natural lesions or transcranial magnetic stimulation of this segment.

In contrast, the frontal components of the fronto-parietal attention network, the frontal and the supplementary eye fields (FEF, SEF), were not activated by dimension changes. This may not be surprising because the FEF and SEF play a prominent role in visuo-spatial shifts of attention and overt eye movements, whereas the cortex along the intraparietal sulcus has been shown to support spatial as well as non-spatial shifts of attention (Coull and Frith, 1998; Wojciulik and Kanwisher, 1998). Thus, changes in the target-defining dimension, which will elicit a shift of attention between visual dimensions, may be expected to lead to activation of the parietal, but not the frontal areas of the fronto-parietal network of attention. The lack of activation in the FEF and SEF further argues against a differential effect of eye movements in the dimension change and no-change conditions.

Left lateral frontopolar cortex, in which we found dimensionchange-related activation in several previous studies (Pollmann et al., 2000a,b; Weidner et al., 2002), did not show a significant dimension-change-related activation in the present experiment. The absence of frontopolar dimension-change-related activation corresponds with the weak dimension change costs in this study. We have found that frontopolar dimension-change-related activation is only observed when dimension changes are stimulus-driven, but not when they are top-down-controlled (Weidner et al., 2002). Possibly, the more complex task demands in the present study, which included both dimension and response changes, led to a higher degree of topdown control-and consequently a reduced involvement of frontopolar cortex. This may have been so especially because, on each trial, attention needed to be switched from the dimension relevant for target detection, color or motion, to the dimension relevant for response, shape (orientation). This possibility, however, needs to be further investigated.

\section{Response change}

Within the group of areas showing response-change-associated signal changes, some areas exhibited a phasic activation, whereas others displayed a longer-lasting activation increase. The most phasic early response-change-related activation was observed in left AIP. A subset of neurons in AIP fires not only in relation to hand movements but is also visually selective, suggesting a role in the visual guidance of hand movements (Grefkes et al., 2002; Taira et al., 1990). In the present context, this may be the finger response compatible with the target triangle's pointing direction. In the current experiment, AIP may have been instrumental in determining the button press response required by the target and in preparing the system for a response change.
Relation of dimension-change- and response-change-elicited activation patterns

We observed a clear-cut separation between brain areas exhibiting dimension-change- and response-change-related activation. This separation supported our assumption that the processes involved in visual dimension changes are different from those involved in response changes. This is remarkable because both kinds of change most likely require a cascade of processing steps. Dimension changes require the change in the target-defining dimension to be detected and, for implementing the putative shift of attentional weight to the new dimension, adjustments to be made in the sensitivity or bias of the visual input structures, which are likely controlled by top-down signals from parietal or frontal areas.

In contrast, response changes require the processing system to link the relevant stimulus change (the target triangle's pointing direction) to a change in the required response and, in turn, selection, preparation, and execution of the new response.

A post-selective response stage account of visual dimension weighting (Cohen and Magen, 1999; Cohen and Feintuch, 2002; Feintuch and Cohen, 2002; Cohen and Shoup, 1997; Mortier et al., 2005; Theeuwes et al., in press; Theeuwes, 1992, 1996) would predict no activation changes in visual input areas to accompany visual dimension changes because perceptual and visual-attentional processes are assumed to remain unchanged. Instead, this account would predict dimension-change-related activation in brain areas supporting response selection and thus an overlap between dimension-change- and response-change-related activation in these areas. Our data do not confirm the hypothesis of a response stage origin of visual dimension change costs: no overlap between dimension-change- and response-change-related activation was observed and, apart from that, no dimension-changerelated activation in brain areas known to support response selection.

\section{Timing differences between dimension- and response-change-related activation}

Dimension changes were followed immediately by a signal increase, relative to dimension repetitions, which peaked $3 \mathrm{~s}$ after stimulus onset in most areas. The earliest response-related activation peaks only occurred $1 \mathrm{~s}$ later. In any case, the data show earlier modulation of the BOLD response by dimension changes than by response changes. Physiological differences in the BOLD response in the various activated brain areas may be a potential confound. This, however, is unlikely as the characteristic time courses-early onset for dimension changes, later onset for response changes-were highly consistent across a wide range of distributed brain areas. In AIP (Fig. 4c) and superior temporal sulcus (Fig. 4d), the characteristic time lag of the response, relative to the dimension, change-related activation was evident even within areas (note, though, that these areas showed no significant signal change related to dimension change).

The compound task required observers to detect the targetdefining singleton feature before they could discriminate the target triangle's pointing direction and select the appropriate response. This sequence of processing steps may be reflected in the time course of the activations. However, the lag of the BOLD signal change for response changes compared to dimension changes, of the order of $1 \mathrm{~s}$, appears incommensurate with the response delay of only $6 \mathrm{~ms}$ for response-change versus response repetition trials. 
Given this, an alternative account for the time lag of the responsechange-related activation may be that the signal change reflects neural processes associated with the execution of the changed, but not that of the unchanged response (rather than being elicited by the discernment of the changed response cue: the target triangle's pointing direction). The average latency for changed responses was $719 \mathrm{~ms}$, which fits well with the observed delay of about $1 \mathrm{~s}$ for response-change-related activation (the temporal resolution of our interpolated signal was $1 \mathrm{~s}$, so the 'true' lag may have been less than $1 \mathrm{~s}$ ). The signal increase may then reflect response facilitation processes which begin after a new response has been executed and continue over several seconds. Particularly, the delayed and longer-lasting signal increases in MI, SI, lateral premotor cortex, and cerebellum may represent a tonic increase in excitability that results when the response change has been carried out and is maintained until the next response change. The average duration from one response change to the next was $5 \mathrm{~s}$, roughly consistent with the plateau phases of the BOLD signal in these areas.

A delayed activation was observed in left middle frontal gyrus and in the left cerebellar hemisphere. Here, the peak of the differential signal increase for response changes versus repetitions was observed after 7-8 s. A similar pattern was found in left lateral premotor cortex, where a second peak at $7 \mathrm{~s}$ followed a first peak at $4 \mathrm{~s}$ post-stimulus onset. These time courses suggest that these areas support processes that take place after a response change has been implemented.

Thus, we found a clear separation between the brain areas activated following dimension changes on the one hand and response changes on the other. The signal modulation elicited by dimension changes becomes manifest earlier than that elicited by response changes, and it is of a more transient nature, while response changes induced more sustained signal increases in several brain areas. Our data support the view that visual dimension changes and response changes involve different processes rather than relying on common high-level (e.g., attentional) change mechanisms.

\section{Interaction between dimension change and response change}

The processes governing dimension and response changes, however, were not completely independent. The behavioral data showed an interaction of dimension and response changes. The fastest responses occurred, unsurprisingly, when neither the targetdefining dimension nor the response changed. Visual dimension changes significantly slowed the latencies for unchanged responses, which were of about the same length as those for changed responses. Conversely, when the target-defining dimension remained the same as on the previous trial, changed responses were significantly slower than unchanged responses. However, coinciding dimension and response changes did not yield an additive slowing of the reactions. This would argue against a serial model, in which perceptual processes, which take longer in the case of a dimension change, are followed by response-related processes, which in turn take longer in the case of a response change. Instead, the data support the idea that repetition of the unchanged target-defining dimension facilitates unchanged responses, whereas a dimension change disrupts this facilitation, removing the 'bias' to select the unchanged over the changed response. This idea is also supported by the functional activation in the parieto-occipital fissure. In this area, repetition of both the dimension and the response on a trial yielded, in absolute terms, the highest signal amplitudes of all conditions. Specifically, the signal was greater for dimension repetitions than for dimension changes on response repetition trials (witness the non-overlap of the standard errors of the means in Fig. 6). Note that higher activation was associated with faster response times, which may reflect the facilitation of unchanged responses when the targetdefining dimension is repeated. There was no such difference when the response changed.

This pattern suggests that, although, statistically, there is no correlation between the two types of change (target-defining dimension, response attribute), the processing system 'assumes' that there is one. If the target dimension (the task attribute that becomes available first) remains unchanged, then the system implicitly assumes that the attribute on which the response is based will stay the same, too; that is, the unchanged response will be facilitated, and there is a cost if the response attribute actually changes. In contrast, if the dimension changes, the system cancels any prior assumption as to the (expected) response attribute and starts processing from scratch. This linkage between dimension and response attribute changes (which is completely unfounded in terms of event statistics) may exist because, for the system, it may be easier to change both task 'expectancies', dimension and response attribute, than to change just one (see Kingstone, 1992; Hillstrom, 2000, for similar arguments).

Thus, we have two sets of brain areas that respond in opposite ways to dimension changes. One set, consisting mainly of posterior visual areas, responds with a signal increase to dimension changes, whereas the other, consisting of the cortex along the parietooccipital fissure, responds with a signal increase to dimension repetition, provided that the response is also repeated. It is likely that the increased dimension-change-related activity characterizes brain areas that are involved in reallocating attention to the new dimension, whereas the increased response on dimension repetition trials characterizes areas involved in maintaining attentional settings for the current dimension (and response). When both the target-defining dimension and the response are repeated, the response may be facilitated.

\section{Conclusions}

Visual dimension changes and response changes were associated with BOLD signal increases in separate sets of brain areas, indicating different neuronal processes to be involved in both types of change. In particular, dimension-change-related activation was observed in visual areas, but not in areas involved in response selection processes. This pattern of activation supports the assumption of a perceptual origin of dimension change costs rather than a response stage account.

Dimension-change-related signal increase began about $1 \mathrm{~s}$ earlier than response-change-related signal increase, suggesting that the former was elicited by perceptual processes, whereas the latter was elicited by response-related or post-response processes.

Although visual dimension changes and response changes rely on different processes, they are not completely independent. The interaction observed in the behavioral and functional imaging data suggests, instead of a serial, an interactive account of dimension and response change costs, in particular: a response facilitation for combined dimension and response repetitions, which may be disrupted when the target-defining dimension changes. 


\section{Acknowledgments}

This study was supported by the Deutsche Forschungsgemeinschaft (DFG grant FOR 309/3-1). We thank an anonymous reviewer for helpful comments on an earlier version of this paper.

\section{Appendix A. Supplementary data}

Supplementary data associated with this article can be found in the online version at doi:10.1016/j.neuroimage.2005.09.013.

\section{References}

Bartels, A., Zeki, S., 2000. The architecture of the colour centre in the human visual brain: new results and a review. Eur. J. Neurosci. 12, $172-193$.

Binkofski, F., Dohle, C., Posse, S., Stephan, K.M., Hefter, H., Seitz, R.J., Freund, H.-J., 1998. Human anterior intraparietal area subserves prehension. A combined lesion and functional MRI activation study. Neurology 50, 1253-1259.

Binkofski, F., Buccino, G., Posse, S., Seitz, R.J., Rizzolatti, G., Freund, H.-J., 1999. A fronto-parietal circuit for object manipulation in man: evidence from an fMRI study. Eur. J. Neurosci. 11, 3276-3286.

Bundesen, C., 1990. A theory of visual attention. Psychol. Rev. 97, $523-547$

Buracas, G.T., Boynton, G.M., 2002. Efficient design of event-related fMRI experiments using M-sequences. Neuroimage 16, 801-813.

Cohen, A., Feintuch, U., 2002. The dimensional-action system: a distinct visual system. In: Prinz, W., Hommel, B. (Eds.), Attention and Performance XIX: Common Mechanisms in Perception and Action. Oxford Univ. Press, Oxford, pp. 587-608.

Cohen, A., Magen, H., 1999. Intra- and cross-dimensional visual search for single feature targets. Percept. Psychophys. 61, 291-307.

Cohen, A., Shoup, R., 1997. Perceptual dimensional constraints on response selection processes. Cogn. Psychol. 32, 128-181.

Corbetta, M., Akbudak, E., Conturo, T.E., Snyder, A.Z., Ollinger, J.M., Drury, H.A., Linenweber, M.R., Petersen, S.E., Raichle, M.E., Essen, D.C.V., Shulman, G.L., 1998. A common network of functional areas for attention and eye movements. Neuron 21, 761-773.

Coull, J.T., Frith, C.D., 1998. Differential activation of right superior parietal cortex and intraparietal sulcus by spatial and nonspatial attention. NeuroImage 8, 176-187.

Duncan, J., 1985. Visual search and visual attention. In: Posner, M.I., Marin, O.S. (Eds.), Attention and Performance XI. Erlbaum, Hillsdale, N.J., pp. $85-106$

Duncan, J., Humphreys, G.W., 1989. Visual search and stimulus similarity. Psychol. Rev. 96, 433-458.

Efron, B., 1977. Bootstrap methods: another look at the jackknife. Ann. Stat. $7,1-26$.

Feintuch, U., Cohen, A., 2002. Visual attention and coactivation of response decisions for features from different dimensions. Psychol. Sci. 13 (4), 361-369

Forman, S.D., Cohen, J.D., Fitzgerald, M., Eddy, W.F., Mintun, M.A., Noll, D.C., 1995. Improved assessment of significant activation in functional magnetic resonance imaging (fMRI): use of a cluster-size threshold. Magn. Reson. Med. 33 (5), 636-647.

Found, A., Müller, H.J., 1996. Searching for unknown feature targets on more than one dimension: investigating a "dimension-weighting" account. Percept. Psychophys. 58, 88-101.

Friston, K.J., Holmes, A.P., Worsley, K.J., Poline, J.B., Frith, C.D., Frackowiak, R.S.J., 1995. Statistical parametric maps in functional imaging: a general linear approach. Hum. Brain Mapp. 2, 189-210.
Gitelman, D.R., Nobre, A.C., Parrish, T.B., LaBar, K.S., Kim, Y.H., Meyer, J.R., Mesulam, M., 1999. A large-scale distributed network for covert spatial attention: further anatomical delineation based on stringent behavioural and cognitive controls. Brain 122 (6), 1093-1106.

Grefkes, C., Weiß, P.H., Zilles, K., Fink, G.R., 2002. Crossmodal processing of object features in human anterior intraparietal cortex: an fMRI study implies equivalencies between humans and monkeys. Neuron 35, $173-184$.

Heinze, H.J., Mangun, G.R., Burchert, W., Hinrichs, H., Scholz, M., Münte, T.F., Gös, A., Scherg, M., Johannes, S., Hundeshagen, H., Gazzaniga, M.S., Hillyard, S.A., 1994. Combined spatial and temporal imaging of brain activity during visual selective attention in humans. Nature 372 , $543-546$.

Hillstrom, A.P., 2000. Repetition effects in visual search. Percept. Psychophys. 62, 800-817.

Holmes, A.P., Friston, K.J., 1998. Generalizability, random effects, and population interference. NeuroImage 7, 754.

Hommel, B., 1998. Event files: evidence for automatic integration of stimulus-response codes. Vis. Cognit. 5, 183-216.

Kingstone, A., 1992. Combining expectancies. Q. J. Exp. Psychol. 44A, 69-104.

Krummenacher, J., Müller, H.J., Heller, D., 2001. Visual search for dimensionally redundant pop-out targets: evidence for parallel-coactive processing of dimensions. Percept. Psychophys. 63, 901-917.

Krummenacher, J., Müller, H.J., Heller, D., 2002a. Visual search for dimensionally redundant pop-out targets: parallel-coactive processing of dimensions is location-specific. J. Exp. Psychol.: Hum. Percept. Perform. 28, 1302-1322.

Krummenacher, J., Müller, H.J., Heller, D., 2002b. Visual search for dimensionally redundant pop-out targets: Redundancy gains in compound tasks. Vis. Cognit. 9, 801-837.

Lepsien, J., Pollmann, S., 2002. Covert reorienting and inhibition of return: an event-related fMRI study. J. Cognit. Neurosci. 14, 127-144.

Liu, T., Slotnick, S.D., Serences, J.T., Yantis, S., 2003. Cortical mechanisms of feature-based attentional control. Cereb. Cortex 13, 1334-1343.

Lockhead, G.R., Gruenewald, P., King, M., 1978. Holistic vs. attribute repetition effects in classifying stimuli. Mem. Cognit. 6, 438-445.

Loftus, G.R., Masson, M., 1994. Using confidence intervals in withinsubject designs. Psychon. Bull. Rev. 4, 476-490.

Lohmann, G., Mueller, K., Bosch, V., Mentzel, H., Hessler, S., Chen, L., von Cramon, D.Y., 2001. Lipsia-A new software system for the evaluation of functional magnetic resonance images of the human brain. Comput. Med. Imaging Graph. 25, 449-457.

Martinez, A., Anllo-Vento, L., Sereno, M.I., Frank, L.R., Buxton, R.B., Dubowitz, D.J., Wong, E.C., Hinrichs, H., Heinze, H.J., Hillyard, S.A., 1999. Involvement of striate and extrastriate visual cortical areas in spatial attention. Nat. Neurosci. 2, 364-369.

Mortier, K., Theeuwes, J., Starreveld, P., 2005. Search for targets within and across dimensions: no top-down modulation. J. Exp. Psychol. Hum. Percept. Perform. 31 (3), 542-557.

Müller, H.J., Krummenacher, J., in press. Locus of dimension weighting: pre-attentive or post-selective? Vis. Cogn.

Müller, H.J., Heller, D., Ziegler, J., 1995. Visual search for singleton feature targets within and across feature dimensions. Percept. Psychophys. 57, $1-17$.

Müller, H.J., Reimann, B., Krummenacher, J., 2003. Stimulus-driven and expectancy-driven effects in dimensional weighting. J. Exp. Psychol.: Hum. Percept. Perform. 29, 1021-1035.

Oldfield, R.C., 1971. The assessment and analysis of handedness: the Edinburgh inventory. Neuropsychologia 9, 97-113.

Pollmann, S., von Cramon, D.Y., 2000. Object working memory and visuospatial processing: functional neuroanatomy analyzed by eventrelated fMRI. Exp. Brain Res. 133, 12-22.

Pollmann, S., Dove, A., von Cramon, D.Y., Wiggins, C.J., 2000a. Eventrelated fMRI: comparison of conditions with varying BOLD-overlap. Hum. Brain Mapp. 9, 26-37.

Pollmann, S., Weidner, R., Müller, H.J., von Cramon, D.Y., 2000b. A 
fronto-posterior network involved in visual dimension changes. J. Cogn. Neurosci. 12, 480-494.

Pollmann, S., Weidner, R., Müller, H.J., von Cramon, D.Y., in press. Neural basis of visual dimension weighting. Vis. Cognit.

Ruge, H., Brass, M., Lohmann, G., von Cramon, D.Y., 2003. Event-related analysis for event types of fixed order and restricted spacing by temporal quantification of trial-averaged fMRI time courses. J. Magn. Res. Imaging 18, 599-607.

Rushworth, M.F., Ellison, A., Walsh, V., 2001. Complementary localization and lateralization of orienting and motor attention. Nat. Neurosci. 4 $656-661$.

Taira, M., Mine, S., Georgopoulos, A.P., Murata, A., Sakata, H., 1990 Parietal cortex neurons of the monkey related to the visual guidance of hand movement. Exp. Brain Res. 83, 29-36.

Talairach, J., Tournoux, P., 1988. Co-Planar Stereotactic Atlas of The Human Brain. Thieme, Stuttgart.

Theeuwes, J., 1992. Perceptual selectivity for color and form. Percept. Psychophys. 51, 599-606.

Theeuwes, J., 1996. Perceptual selectivity for color and form: on the nature of the interference effect. In: Kramer, A.F., Coles, M.G.H. (Eds.), Converging Operations in The Study of Visual Selective Attention. American Psychological Association, Washington, DC, pp. 297-314.

Theeuwes, J., Reimann, B., Mortier, K., in press. Visual search for featural singletons: no top-down modulation, only bottom-up priming. Vis. Cognit.

Weidner, R., Pollmann, S., Müller, H.J., von Cramon, D.Y., 2002. Topdown controlled visual dimension weighting: an event-related fMRI study. Cereb. Cortex 12, 318-328.

Wojciulik, E., Kanwisher, N., 1998. The generality of parietal involvement in visual attention. Neuron 23, 747-764

Yantis, S., Schwarzbach, J., Serences, J.T., Carlson, R.L., Steinmetz, M.A., Pekar, J.J., Courtney, S.M., 2003. Transient neural activity in human parietal cortex during spatial attention shifts. Nat. Neurosci. 5, $995-1002$.

Yousry, T.A., Schmid, U.D., Alkadhi, H., Schmidt, D., Peraud, A., Buettner, A., Winkler, P., 1997. Localization of the motor hand area to a knob on the precentral gyrus. Brain 120, 141-157. 